\title{
Magnetic field induced competing phases in spin-orbital entangled Kitaev magnets
}

\author{
Li Ern Chern $\odot,{ }^{1}$ Ryui Kaneko $\odot,{ }^{2}$ Hyun-Yong Lee $\odot,{ }^{2}$ and Yong Baek Kim ${ }^{1,3,4,5}$ \\ ${ }^{1}$ Department of Physics, University of Toronto, Toronto, Ontario, Canada M5S 1A7 \\ ${ }^{2}$ Institute for Solid State Physics, University of Tokyo, Kashiwa, Chiba 277-8581, Japan \\ ${ }^{3}$ Perimeter Institute for Theoretical Physics, Waterloo, Ontario, Canada N2L $2 Y 5$ \\ ${ }^{4}$ Canadian Institute for Advanced Research/Quantum Materials Program, Toronto, Ontario, Canada M5G $1 Z 8$ \\ ${ }^{5}$ School of Physics, Korea Institute for Advanced Study, Seoul 130-722, Korea
}

(Received 30 September 2019; revised manuscript received 19 November 2019; published 6 January 2020)

\begin{abstract}
There has been a great interest in magnetic field induced quantum spin liquids in Kitaev magnets after the discovery of neutron scattering continuum and half-quantized thermal Hall conductivity in the material $\alpha-\mathrm{RuCl}_{3}$. In this work, we provide a semiclassical analysis of the relevant theoretical models, which enable us to treat large system sizes approximating the thermodynamic limit. We find a series of competing magnetic orders with fairly large unit cells at intermediate magnetic fields, which are mostly missed by previous studies. We show that quantum fluctuations are typically strong in these large unit cell orders, while the magnetic excitations, magnons, have a dispersion that resembles a scattering continuum. The huge quantity of magnon bands with finite Chern numbers also gives rise to an unusually large thermal Hall conductivity. Given the highly frustrated nature of the spin model, the large unit cell orders are likely to melt into the putative spin liquid in the quantum limit. Our work provides an important basis for a thorough investigation of emergent spin liquids and competing phases in Kitaev magnets.
\end{abstract}

DOI: 10.1103/PhysRevResearch.2.013014

\section{INTRODUCTION}

Discovery of quantum spin liquids [1,2] with emergent quasiparticles has been an important subject in modern condensed matter physics. This serves as an ultimate test of our understanding of highly quantum entangled phases in interacting electron systems. Recent research has invested tremendous effort on a number of materials with strong spin-orbit coupling $[3,4]$, which leads to intriguing bonddependent exchange interactions between spin-orbital entangled pseudospin-1/2 moments. These studies are largely motivated by the exact solution of the Kitaev honeycomb model [5]. The Kitaev interaction is naturally present in the systems with $4 d / 5 d$ transition metal elements [6], such as honeycomb/hyperhoneycomb iridates [7-9] and $\alpha-\mathrm{RuCl}_{3}$ [10]. However, other exchange interactions are present too [11], which often lead to magnetically ordered ground states instead of the desired quantum spin liquid [12-15]. Hence, much effort has been spent to suppress the magnetic orders and gain access to the possible spin liquid phases.

Over the past few years, great experimental progress has been achieved in $\alpha-\mathrm{RuCl}_{3}$. At zero magnetic field, this material orders magnetically in the zigzag (ZZ) order $[14,15]$. Upon the application of an external field, neutron scattering experiments [16-18] find an intermediate window of fields

Published by the American Physical Society under the terms of the Creative Commons Attribution 4.0 International license. Further distribution of this work must maintain attribution to the author(s) and the published article's title, journal citation, and DOI. before the system enters the polarized state, where sharp magnon modes are absent but a scattering continuum appears instead. Under a [111] field (perpendicular to the honeycomb plane), the measured thermal Hall conductivity above the ordering temperature $T_{\mathrm{N}} \approx 7 \mathrm{~K}$ follows the predicted trend of itinerant Majorana fermions in the pure Kitaev model [19]. When the field is tilted away from the [111] direction by $45^{\circ}$ and $60^{\circ}$, half-quantized thermal Hall conductivity is observed [20]. These observations raise the hope that the paramagnetic state in the intermediate field regime may be the sought-after chiral spin liquid with Majorana edge modes.

Theoretical models for $\alpha-\mathrm{RuCl}_{3}$ include substantial $\mathrm{Ki}$ taev and symmetric anisotropic $\Gamma$ interactions, both strongly dependent on the bond directions, with additional small exchanges such as the nearest neighbor Heisenberg $J$, the third nearest neighbor Heisenberg $J_{3}$, and the anisotropic $\Gamma^{\prime}$ [21-23], on the honeycomb lattice. Previous analyses are largely done on quantum models with small system sizes (typically a 24-site cluster) via exact diagonalization (ED) [24-28] or in quasi-one-dimensional limit via density matrix renormalization group (DMRG) [26,27,29], with varying degree of complexity. For example, a recent work [27] on the $K \Gamma \Gamma^{\prime}$ model in an external magnetic field suggests that it allows an intermediate spin liquid phase continuously connected to the pure Kitaev model between the low-field $\mathrm{ZZ}$ order and high-field polarized state.

In this article, we investigate the possible competing phases in the classical $K \Gamma \Gamma^{\prime}$ model under a [111] magnetic field for large system sizes. The purpose is to critically examine what kind of competing phases may be present and how these phases may be related to potential spin liquids in the quantum model. Rather surprisingly, we find a series of competing 
magnetic orders with large unit cells in the intermediate field regime. In particular, in the $K \Gamma \Gamma^{\prime}$ model with small $\Gamma^{\prime}$, the ground state in the zero field limit is the $\mathrm{ZZ}$ order, which is consistent with previous experiments and theoretical calculations. Upon increasing the field, the $\mathrm{ZZ}$ order is replaced by a series of magnetically ordered phases with 8-, 18-, 32-, 50-, 70-, and 98-site unit cells before the system enters the polarized state (see Fig. 2). Hence, the magnetic field reveals a series of competing orders, which form an intermediate region in the phase diagram. Most of these large unit cell orders had not been identified in previous works.

We compute the zero point quantum fluctuations for these magnetic orders and estimate the reduction of the size of the local moments. We find that quantum fluctuations are strong in the large unit cell orders so that the renormalized local moment is only about $50 \%$ of the full magnitude on average. The flat and dense spin wave spectra in the large unit cell orders, in particular the 70- and 98-site orders, essentially look like continua of spin excitations. Furthermore, we calculate the thermal Hall conductivity due to magnons in some of the large unit cell orders and find that it is as large as that observed experimentally at low temperatures. While strong quantum fluctuations are present and hence it is likely for the series of competing phases to turn into spin liquids in the quantum limit, it is also evident that previous theoretical studies on quantum models with small system sizes [24-29] cannot resolve many of these large unit cell orders. Therefore, in future analyses of such quantum models, it will be important to understand the role of quantum fluctuations in the large unit cell orders unveiled in the current work. Our findings demonstrate the possibility of novel and exotic ordering patterns in spin-orbital entangled Kitaev magnets, which provide an important basis for further investigations of the origin and the nature of quantum spin liquids that they may host.

\section{MODEL}

We investigate the nearest neighbor $K \Gamma \Gamma^{\prime}$ model on the honeycomb lattice in a [111] magnetic field $\mathbf{h}$,

$$
\begin{aligned}
H & =\sum_{\lambda=x, y, z} \sum_{\langle i j\rangle \in \lambda}\left[K S_{i}^{\lambda} S_{j}^{\lambda}+\Gamma\left(S_{i}^{\mu} S_{j}^{\nu}+S_{i}^{\nu} S_{j}^{\mu}\right)\right. \\
& \left.+\Gamma^{\prime}\left(S_{i}^{\mu} S_{j}^{\lambda}+S_{i}^{\lambda} S_{j}^{\mu}+S_{i}^{\nu} S_{j}^{\lambda}+S_{i}^{\lambda} S_{j}^{\nu}\right)\right]-\mathbf{h} \cdot \sum_{i} \mathbf{S}_{i},
\end{aligned}
$$

where $K$ is the Kitaev interaction, $\Gamma$ and $\Gamma^{\prime}$ are off-diagonal spin exchanges, $(\lambda, \mu, v)$ is a cyclic permutation of $(x, y, z)$, and the field $\mathbf{h}=h(1,1,1) / \sqrt{3}$. We have also assumed an isotropic $g$ tensor. In (1), $h$ actually carries a factor of $S$ but for notational simplicity we will just write $h$ in units of the Kitaev interaction, for instance $h=0.1|K|$ instead of $h=0.1|K| S$, in the rest of this article.

In the experimentally relevant parameter regime, $K<0$ and $\Gamma>0$ are large while $\Gamma^{\prime}<0$ is small. In contrast to many of the previous studies [24-30], we investigate the classical limit of this model, that is, by treating the spins $\mathbf{S}_{i}=\left(S_{i}^{x}, S_{i}^{y}, S_{i}^{z}\right)$ in (1) as three-dimensional vectors of fixed magnitude $\left|\mathbf{S}_{i}\right|=S$ for all $i$. We use simulated annealing to determine the ground-state spin configuration of the system.

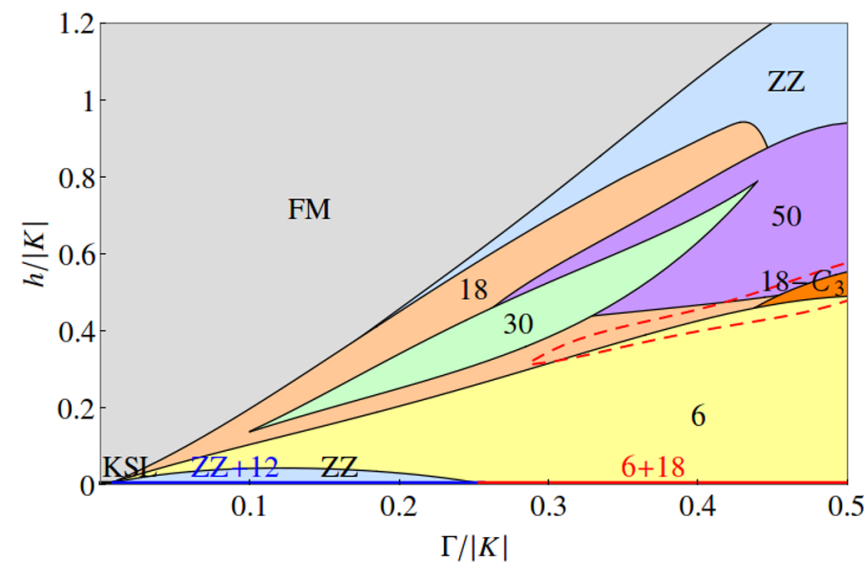

FIG. 1. Classical phase diagram of the $K \Gamma$ honeycomb model in a [111] magnetic field $h$. Ferromagnetic Kitaev interaction $K=-1$ is assumed. Each of the integers indicates the total number of sublattices in a unit cell of the corresponding magnetic order, while + indicates degeneracy. KSL denotes the extensively degenerate manifold of the Kitaev model, which only exists in the Kitaev limit $(\Gamma, h)=(0,0)$. The ground state in the parameter region enclosed by the red dashed line is likely an incommensurate order which exhibits domains of 18 and $18-C_{3}$.

Details of the simulated annealing calculation can be found in Appendix A.

\section{RESULTS}

\section{A. Phase diagrams}

We first consider the $K \Gamma$ model by setting $\Gamma^{\prime}=0$ in (1), with a ferromagnetic Kitaev interaction $K=-1$. We explore $\Gamma \in[0,0.5], h \in[0,1.2]$ and map out the phase diagram, as shown in Fig. 1. Apart from the extensively degenerate Kitaev limit $(\Gamma, h)=(0,0)$, we find that the vast majority of the parameter space favors particular magnetic orders. All these ordered phases, except the zigzag (ZZ) order and the ferromagnet (FM), are labeled by the number of sites contained in their respective magnetic unit cells.

In the zero field limit, the degeneracy of the Kitaev manifold is lifted as the ZZ order and a 12-site order (a 6-site order and an 18-site order) are selected at small (intermediate) $\Gamma$. These two phases have exactly the same energy at $h=0$, but the ZZ order or the 6 -site order is preferred once $h \neq 0$. However, the 18-site order reemerges at higher fields and replaces the 6-site order as the ground state. Tracing back to the parameter region with small $\Gamma$ and $h$, we see that the ZZ, 6 -site, 12-site, and 18-site orders are continuously connected to the Kitaev limit. The 6-site order (the 18 -site order) was first reported in Ref. [31] (Ref. [32]) and termed the X phase (the diluted star phase). At sufficiently large values of $\Gamma$ and $h$, even larger cluster ordering patterns like the 30 -site and 50 -site orders are stabilized. There is also an 18-site order with $C_{3}$ symmetry, which we label by $18-C_{3}$ to distinguish it from the previous 18 -site order as they are described by different arrangements of spins on the honeycomb lattice.

Next, we set $\Gamma^{\prime}=-0.02$ and map out the phase diagram within the same ranges of $\Gamma$ and $h$, as shown in Fig. 2. The 


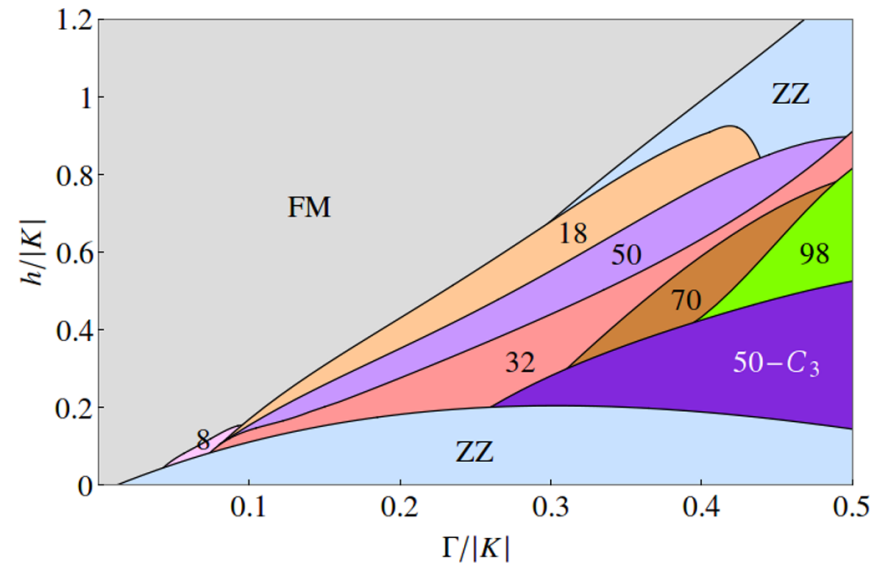

FIG. 2. Classical phase diagram of the $K \Gamma \Gamma^{\prime}$ honeycomb model in a [111] magnetic field $h$. Ferromagnetic Kitaev interaction $K=-1$ is assumed. $\Gamma^{\prime}$ is fixed to be -0.02 . Each of the integers indicates the total number of sublattices in a unit cell of the corresponding magnetic order.

addition of such a small $\Gamma^{\prime}$ term to the $K \Gamma$ model alters the phase diagram quite significantly. The degenerate manifold in the Kitaev limit and its neighborhood are replaced by the FM phase. The $\mathrm{ZZ}$ order is stabilized over a large portion of the parameter space at zero [33] and low fields. Once again, we find at intermediate fields several large cluster ordering patterns, a 32 -site order, a 70 -site order, a 98 -site order, and a 50 -site order with $C_{3}$ symmetry, which we label by $50-C_{3}$. Finally, the strong $\Gamma$ high field regime of the phase diagram displays some similarities to the $\Gamma^{\prime}=0$ case, where the same 50-site, 18-site, and ZZ order are the lowest energy spin configurations, before the system becomes a FM.

Details of the magnetic orders (the real space spin configurations and the static spin structure factors, etc.) that show up in the phase diagrams Figs. 1 and 2, from the four-sublattice $\mathrm{ZZ}$ order to the 98-site order, can be found in the Supplemental Material [34]. We make some qualitative observations as follows. Firstly, stronger $\Gamma$ interaction stabilizes magnetic orders with larger unit cells. This is true for both zero and finite $\Gamma^{\prime}$. We expect that ordering patterns with even larger unit cells than those mentioned above may appear if $\Gamma$ is further increased beyond 0.5 . Secondly, the large unit cell orders, like the 70-site and 98-site orders, are closely competing in the parameter region where they are stabilized. The difference in energy is typically $10^{-3}$ to $10^{-4}$ of the energies of these orders. Thirdly, the magnetic orders can be classified into two categories, one with an inversion symmetry and the other with a three fold rotational symmetry. The $\mathrm{ZZ}$ order and the magnetic orders labeled by numbers fall into the former, while the magnetic orders labeled by numbers appended with $-C_{3}$ fall into the latter. More details can be found in Ref. [34].

\section{B. Magnetization}

The proposed spin model for the material $\alpha-\mathrm{RuCl}_{3}$ is parametrized by dominant $K$ and $\Gamma$ exchanges, with $K<0$ and $\Gamma \approx-K / 2$, plus some small additional interactions like $\Gamma^{\prime}, J$ and $J_{3}$, where $J\left(J_{3}\right)$ is the (third) nearest neighbor Heisenberg exchange [21-23]. Therefore, in the phase dia-

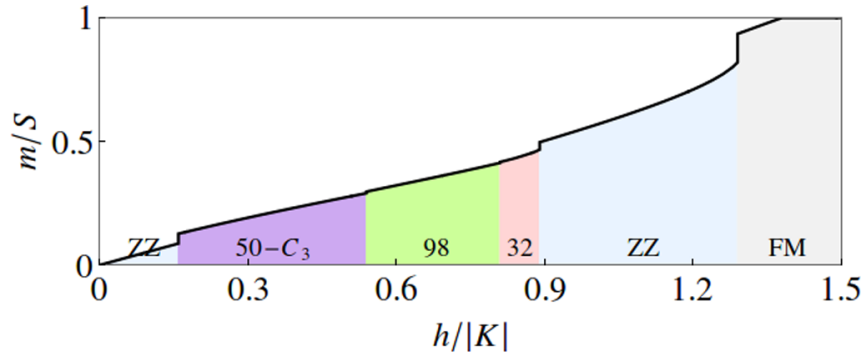

(a)

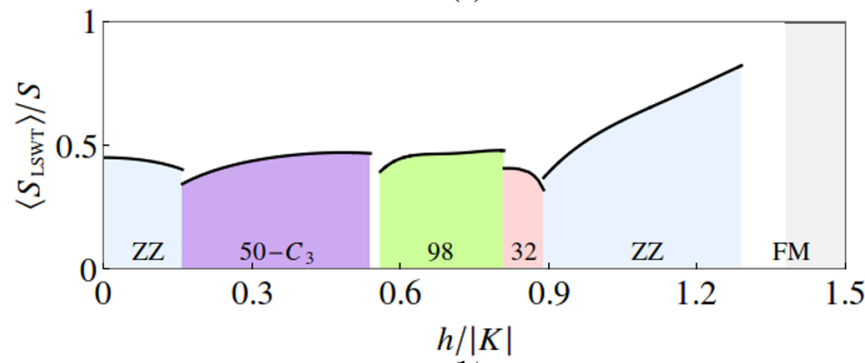

(b)

FIG. 3. (a) The magnetization along the field direction, with the parametrization $\left(K, \Gamma, \Gamma^{\prime}\right)=(-1,0.5,-0.02)$ relevant to the material $\alpha-\mathrm{RuCl}_{3}$. (b) The averaged renormalization of ordered moments when quantum fluctuations are taken into account via the linear spin wave theory with $S=1 / 2$, using the same parametrization as in panel (a), at zero temperature. Blank regions indicate that the spin-wave Hamiltonian is not positive definite.

gram Fig. 2 of the $K \Gamma \Gamma^{\prime}$ model in a [111] magnetic field, we take a cut along $\Gamma=0.5$ and plot the magnetization $m=\mathbf{S} \cdot \hat{\mathbf{h}}$ as a function of the field $h$, as shown in Fig. 3(a). The magnetization increases monotonically with the field and jumps at the phase transitions. The discontinuities are not very obvious at the transitions between the large unit cell orders, but are significant when the system enters to (exit from) a large unit cell order from (to) ZZ and from ZZ to FM. This suggests the difficulty of detecting phase transitions at intermediate fields by inspecting the magnetization, if they exist at all in the quantum model.

\section{Linear spin wave theory}

As a first approach to study the effect of quantum fluctuations on the classical orders, we apply the linear spin wave theory $[35,36]$ to calculate the reduction of ordered moments in the zero-temperature limit. For simplicity, we assume the same underlying magnetic orders and do not consider how the classical phase diagram may be changed due to quantum correction to the energy because there are too many competing phases. Details of the linear spin wave calculation can be found in Appendix B. In Fig. 3(b), we plot the average fraction of spins achieved in the linear spin wave theory with $S=1 / 2$ as a function of the field

$$
\frac{\left\langle S_{\mathrm{LSWT}}\right\rangle}{S}=1-\frac{\sum_{i}\left\langle b_{i}^{\dagger} b_{i}\right\rangle / N_{\text {site }}}{S},
$$

where $b_{i}^{\dagger}$ is the magnon creation operator at site $i$ and $N_{\text {site }}$ is the total number of sites in the system. Blank regions indicate that the spin wave Hamiltonian is not positive 


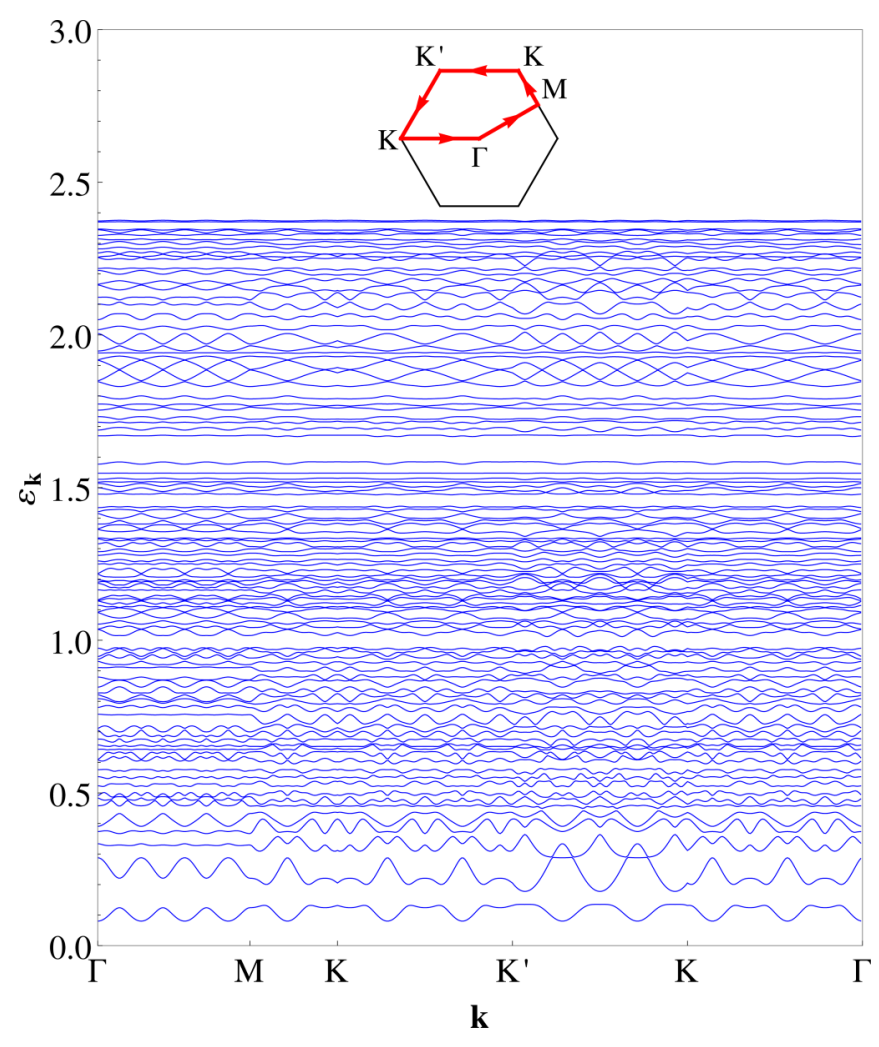

FIG. 4. Spin wave dispersion of the 98 -site order. Plotted in units of $|K| S$, with the parametrization $\left(K, \Gamma, \Gamma^{\prime}\right)=(-1,0.5,-0.02)$ and at the field $h=0.6$. The inset shows the path traveled in the first Brillouin zone of the honeycomb lattice.

definite at one or more momenta; i.e., the lowest magnon band becomes gapless. At low and intermediate fields $h \lesssim 1$, the average reduction of ordered moments is about $50 \%$ of the full magnitude $S$, hinting at strong quantum fluctuations. At high fields $h \gtrsim 1,\left\langle S_{\mathrm{LSWT}}\right\rangle / S$ increase monotonically with $h$ in the $\mathrm{ZZ}$ phase, but the spin wave spectrum becomes gapless throughout the region $h \in[1.29,1.37]$, where the system is in the FM phase with the spins not completely aligning with the [111] field [see Fig. 3(a)]. The physical origin of this phase will be discussed in details later. Finally, for $h>1.37$, the system enters the fully polarized state and $\left\langle S_{\mathrm{LSWT}}\right\rangle / S=1$ achieves saturation.

The spin wave dispersion of a (very) large unit cell order typically appears flat and dense. As an example, we show the spin wave dispersion of the 98-site order along certain high-symmetry directions in the first Brillouin zone of the honeycomb lattice in Fig. 4.

\section{Thermal Hall conductivity}

We calculate the thermal Hall conductivity due to magnons [37-39],

$$
\kappa_{x y}=-\frac{k_{\mathrm{B}}^{2} T}{\hbar V} \sum_{\mathbf{k}} \sum_{n=1}^{\mathcal{N}}\left\{c_{2}\left[f_{\mathrm{BE}}\left(\varepsilon_{\mathbf{k}, n}\right)\right]-\frac{\pi^{2}}{3}\right\} \Omega_{\mathbf{k}, n} .
$$

Details of the calculation can be found in Appendix C. Expressing the field in (1) as $\mathbf{h}=g \mu_{\mathrm{B}} \mu_{0} \mathbf{H}$, assuming the $g$ factor $g=2.3[36,40]$ and the magnitude of the Kitaev interaction $|K| \approx 80 \mathrm{~K}[19,21]$, the field $\mu_{0} H=12 \mathrm{~T}$ used in the experiment of Ref. [19] roughly corresponds to $h=$ 0.23 . At this field and with the parametrization $\left(K, \Gamma, \Gamma^{\prime}\right)=$ $(-1,0.5,-0.02)$, the system is in the $50-C_{3}$ order. We plot the thermal Hall conductivity $\kappa_{x y}$ as a function of temperature $T$, as shown in Fig. 5(a). We show only data below $T_{\mathrm{c}} \approx 11 \mathrm{~K}$, defined as the temperature at which $\left\langle S_{\mathrm{LSWT}}\right\rangle / S$ drops to zero, i.e., the magnetic order is destroyed by thermal fluctuations [see Fig. 5(c)]. We find that $\kappa_{x y}$ is close to zero but slightly negative at $10 \mathrm{~K}$. It gradually develops a positive value as $T$ decreases and peaks at $5 \mathrm{~K}$ before diminishing again as $T \rightarrow 0$. Although the magnon thermal Hall conductivity does not develop a wide plateau, the maximum value of $\kappa_{x y} / T$ is about $0.3 \times 10^{-3} \mathrm{~W} / \mathrm{K}^{2} \mathrm{~m}$, which is of the same order of magnitude as the half-quantized value $0.826 \times 10^{-3} \mathrm{~W} / \mathrm{K}^{2} \mathrm{~m}$ measured in Ref. [20].

We also calculate the thermal Hall conductivity for another large unit cell order, the 32-site order, at the field $h=0.82$ (which would roughly correspond to $\mu_{0} H=43 \mathrm{~T}$ ) and with the same parametrization, as shown in Fig. 5(b). This time $T_{\mathrm{c}} \approx 6 \mathrm{~K}$ [see Fig. $5(\mathrm{c})$ ] and $\kappa_{x y}$ is negative. Starting from zero temperature, $\kappa_{x y}$ grows in magnitude as $T$ increases, and reaches $-0.9 \times 10^{-3} \mathrm{~W} / \mathrm{Km}$ at $6 \mathrm{~K}$. The trend and the magnitude of $\kappa_{x y}$ are similar to those reported in Ref. [19] at lower fields $\left(\mu_{0} H=6,12\right.$ and $\left.15 \mathrm{~T}\right)$. Hence, the opposite signs of $\kappa_{x y}$ may indicate the presence of different magnetic orders.

\section{E. Frustrated ferromagnet}

We notice that there is a window of $h$ where the system is a FM but not fully polarized, i.e., the spins align uniformly but not in the direction of the [111] field. Such a phase is also stabilized in the high field regime at other parametrizations $\left(K, \Gamma, \Gamma^{\prime}\right)$ including the $K \Gamma$ model, and the width of the window is usually larger for stronger $\Gamma$. The spin wave spectrum is also gapless throughout this phase. In the following, we attempt to derive some analytical understanding of why this situation occurs.

We start from the $K \Gamma$ model with $K<0, \Gamma>0$, and $\Gamma^{\prime}=0$ in (1). Assuming a FM state, that is, $\mathbf{S}_{i}=\mathbf{S}$ for all sites $i$, the Hamiltonian reduces to

$$
H=N \mathbf{S}^{\mathrm{T}}\left(H_{K}+H_{\Gamma}\right) \mathbf{S}-2 N \mathbf{h} \cdot \mathbf{S},
$$

with the matrices

$$
H_{K}=\left(\begin{array}{ccc}
K & 0 & 0 \\
0 & K & 0 \\
0 & 0 & K
\end{array}\right), \quad H_{\Gamma}=\left(\begin{array}{ccc}
0 & \Gamma & \Gamma \\
\Gamma & 0 & \Gamma \\
\Gamma & \Gamma & 0
\end{array}\right),
$$

and $N$ being the total number of unit cells. The Kitaev interaction becomes "isotropic" in the FM state, behaving like the Heisenberg interaction. The $\Gamma$ interaction still appears quite anisotropic at this stage, but a change of basis will bring it to a simpler and more illuminating form. Switching from the cubic $x y z$ coordinates to the crystallographic $a b c$ coordinates, where the $a, b$, and $c$ axes point in the directions [11 $]$, [1110], 


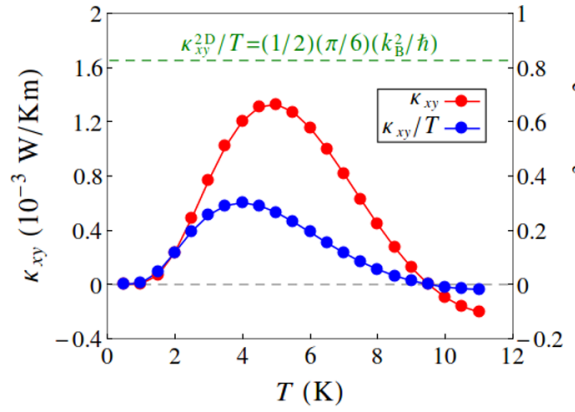

(a)

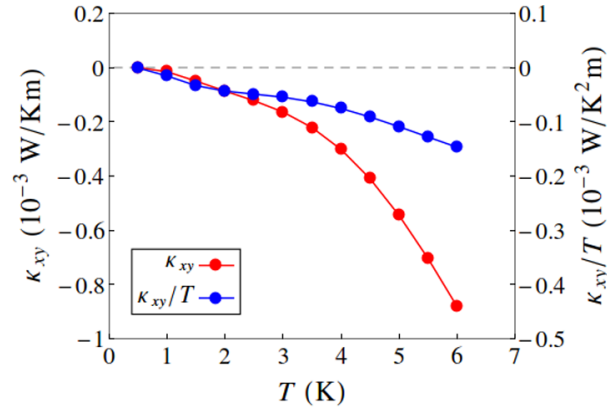

(b)

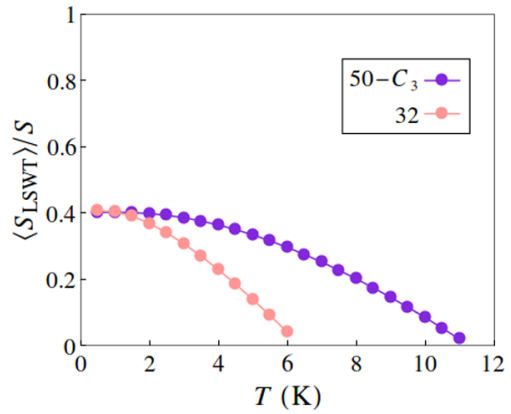

(c)

FIG. 5. Thermal Hall conductivity due to magnons as a function of temperature. (a) The $50-C_{3}$ order at the field $h=0.23$ and (b) the 32 -site order at the field $h=0.82$, both with the parametrization $\left(K, \Gamma, \Gamma^{\prime}\right)=(-1,0.5,-0.02)$. We also indicate in panel (a) the half-quantized thermal Hall conductivity $\kappa_{x y}^{2 \mathrm{D}}=\kappa_{x y} d=(1 / 2)(\pi / 6)\left(k_{\mathrm{B}}^{2} / \hbar\right) T$, or $\kappa_{x y} / T \approx 0.826 \times 10^{-3} \mathrm{~W} / \mathrm{K}^{2} \mathrm{~m}$ with the inter-plane distance $d=5.72 \AA$ $[19,20]$. (c) The averaged renormalization of ordered moments at finite temperatures calculated from the linear spin wave theory, for the two magnetic orders in panels (a) and (b). The Kitaev interaction is assumed to have a magnitude of $80 \mathrm{~K}$.

and [111] respectively [25,41], the spin is given by

$$
\tilde{\mathbf{S}}=\left(\begin{array}{l}
S^{a} \\
S^{b} \\
S^{c}
\end{array}\right)=\left(\begin{array}{ccc}
\frac{1}{\sqrt{6}} & \frac{1}{\sqrt{6}} & -\sqrt{\frac{2}{3}} \\
-\frac{1}{\sqrt{2}} & \frac{1}{\sqrt{2}} & 0 \\
\frac{1}{\sqrt{3}} & \frac{1}{\sqrt{3}} & \frac{1}{\sqrt{3}}
\end{array}\right)\left(\begin{array}{l}
S^{x} \\
S^{y} \\
S^{z}
\end{array}\right)=R \mathbf{S} .
$$

In the $a b c$ basis, the Kitaev interaction $\tilde{H}_{K}=R H_{K} R^{\mathrm{T}}=H_{K}$ remains the same, while the $\Gamma$ interaction assumes the form of an XXZ model

$$
\tilde{H}_{\Gamma}=R H_{\Gamma} R^{\mathrm{T}}=\left(\begin{array}{ccc}
-\Gamma & 0 & 0 \\
0 & -\Gamma & 0 \\
0 & 0 & 2 \Gamma
\end{array}\right) .
$$

We can then analyze (4) in the $a b c$ basis term by term. It can be shown analytically that the energy of the classical Kitaev model is $K|\mathbf{S}|^{2}$ per unit cell [42]. Thus, any FM phase will

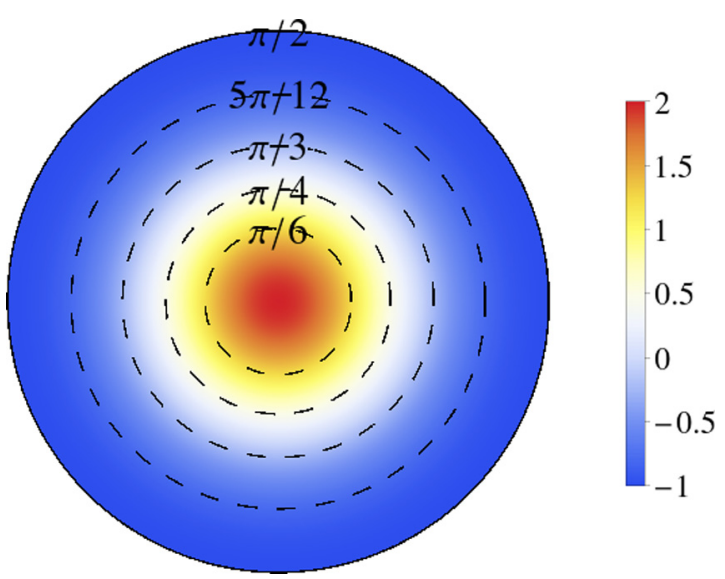

FIG. 6. Energy of the $\Gamma$ interaction in the FM phase. Plotted on the northern hemisphere, where the numbers on the circles indicate the zenith angle $\theta$ defined through $\sqrt{\left(S^{a}\right)^{2}+\left(S^{b}\right)^{2}}=\sin \theta, S^{c}=\cos \theta$. The north pole $\theta=0$ at the center corresponds to $S^{c}=1$, while the equator $\theta=\pi / 2$ intersects the $a b$ plane. The energy possesses an azimuthal or $U(1)$ symmetry. The energy on the southern hemisphere is given by the same density plot. minimize the energy of the $K$ term. On the other hand, the $\Gamma$ term attains maximum (minimum) when the spin points along (lies on) the $c$ axis ( $a b$ plane). The energy profile of the $\Gamma$ term is shown in Fig. 6.

Suppose that the field $\tilde{\mathbf{h}}=(0,0, h)$ is along the $c$ axis or the [111] direction. The $h$ term wants to align the spin with the $c$ axis, but this will be costly in energy for the $\Gamma$ term. The competition between $\Gamma$ and $h$ tilts the spin away from the $c$ axis. Therefore, such a FM phase can be stabilized between the fully polarized state and some other orders, e.g., ZZ and 18 , in the high field regime. In contrast, if the field is along any of the in-plane directions, then all the $K, \Gamma$, and $h$ terms in (4) can be minimized simultaneously.

The effective Hamiltonian (4) describing the FM but not fully polarized phase has a $U(1)$ or azimuthal symmetry, which is manifest in the crystallographic $a b c$ basis. When the spins choose to align in a particular direction among the ground-state manifold, this continuous symmetry is spontaneously broken. Gapless excitation is then guaranteed by Nambu-Goldstone theorem, which is why the lowest magnon band touches the zero energy.

Now let us consider the $K \Gamma \Gamma^{\prime}$ model. A finite $\Gamma^{\prime}$ term acts similarly as $\Gamma$. One can easily show that, assuming a FM state, $H_{\Gamma^{\prime}}$ has the same structure as $H_{\Gamma}$ in (5). Thus, a small $\Gamma^{\prime}<0$ $\left(\Gamma^{\prime}>0\right)$ weakens (enhances) the effect of $\Gamma$. A similar FM but not fully polarized phase due to the presence of a large $\Gamma$ interaction in the $J K \Gamma J_{3}$ model under a [001] field was also found and discussed in Ref. [43].

\section{DISCUSSION}

Classcially, the pure Kitaev model is extremely sensitive to an external magnetic field. It is polarized whenever the field $h \neq 0$. From the result of our simulated annealing calculation, a finite $\Gamma$ interaction on top of $K$ gives rise to a multitude of ordered phases, many of which possess fairly large magnetic unit cells, at finite fields. As $\Gamma$ increases, the window of these nontrivial magnetic orders becomes wider, and the system becomes polarized at greater value of $h$. Thus, the combination of $\Gamma$ and $h$ effects like a prism that produce a rich and colorful phase diagram. Adding a small $\Gamma^{\prime}$ term stabilizes even larger 
cluster magnetic orders at intermediate fields. We successfully demonstrate that the $K \Gamma \Gamma^{\prime}$ honeycomb model is a playground for many exotic field induced magnetic orders, not simply the zigzag (ZZ) order and the polarized state, as largely perceived in the past.

We discuss the implications of these large unit cell orders. First of all, the size of the system has to be sufficiently large to host them. If the system is smaller than or incommensurate with the magnetic order, the ground-state spin configuration may appear like a disordered state. This calls for a serious reconsideration of the results from quantum calculations on small systems where finite-size effect can be important, such as ED on the 24-site cluster [27] and iDMRG on the cylinder geometry [29], which report a quantum spin liquid ground state. The large unit cell magnetic orders found in this work cannot be captured by these and similar computations [24-26,28] on quantum models with small system sizes. Nevertheless, the possibility of a quantum spin liquid still exists, especially in the vicinity of the large unit cell orders where quantum fluctuations are strong. The large unit cell orders are very close in energy in the parameter region where they are stabilized. In addition, the average spin wave correction to the ordered moments in the large unit cell orders for a representative parametrization of $\alpha-\mathrm{RuCl}_{3}$ is found to be more than $50 \%$. One can imagine that quantum fluctuations may melt these competing orders and promote a spin liquid state, but we will not know whether this is true until the magnetic orders are explicitly taken into account in the quantum model. If the large unit cell orders (partially) survive under quantum fluctuations, the magnon bands are typically flat and very close to each other such that they appear like the excitation continuum seen in inelastic neutron scattering experiments, which is often interpreted as fractionalized excitations in a quantum spin liquid [16-18,44,45]. The resulting two magnon excitations will also form a very broad continuum at low energies. Moreover, we calculate the magnon thermal Hall conductivity for two of the large unit cell orders and show that it resembles the trend and/or the magnitude as that measured in experiments $[19,20]$ below the ordering temperature. In contrast, as computed in Ref. [36], the magnon thermal Hall conductivity in the $\mathrm{ZZ}$ order is in general quite small in magnitude.

We also discover the existence of a ferromagnetic (FM) but not fully polarized state at high fields in the $K \Gamma \Gamma^{\prime}$ model with zero or small $\Gamma^{\prime}$, which can be understood through the competition between the $\Gamma$ and $h$ terms. Here $K<0$ and $\Gamma>0$ are assumed. While the field always wants to orient the spins in its direction, the $\Gamma$ interaction is only minimized (maximized) when the spins are all lying on the $a b$ plane (pointing along the $c$ axis). This may explain why the system is more prone to polarization when the tilting angle of the field from the [111] direction is larger. This also suggests that frustration is stronger (weaker) when the field is along (in) the $c$ axis ( $a b$ plane). For instance, the simulated annealing calculation on the classical $J K \Gamma J_{3}$ honeycomb model in an in-plane field [31] with the parametrization $\left(J, K, \Gamma, J_{3}\right)=$ $(-0.035,-1,0.5,0.035)$ only yields the 6-site order (termed the $\mathrm{X}$ phase) at intermediate fields, between the $\mathrm{ZZ}$ order at low fields and the polarized state at high fields, thus leading to a relatively simple phase diagram.

\section{ACKNOWLEDGMENTS}

We thank Hae-Young Kee, Jacob Gordon, Jonathan Cookmeyer, and Kyusung Hwang for useful discussions. We also thank Lukas Janssen for bringing our attention to Ref. [43]. L.E.C. is grateful to Andrei Catuneanu, Panagiotis Peter Stavropoulos, and Sopheak Sorn for teaching him the particulars of the simulated annealing calculation. R.K. was supported by MEXT as "Priority Issue on Post-K Computer" (Creation of New Functional Devices and High-Performance Materials to Support Next-Generation Industries). H.-Y.L. was supported by MEXT as "Exploratory Challenge on PostK Computer" (Frontiers of Basic Science: Challenging the Limits). Y.B.K. was supported by the Killam Research Fellowship from the Killam Foundation, the NSERC of Canada, and the Center for Quantum Materials at the University of Toronto. Most of the computations were performed on the Niagara supercomputer at the SciNet HPC Consortium [46]. SciNet is funded by the Canada Foundation for Innovation, the Government of Ontario, Ontario Research Fund-Research Excellence, and the University of Toronto. Part of the computations were performed at the Supercomputer Center, ISSP, University of Tokyo.

\section{APPENDIX A: DETAILS OF SIMULATED ANNEALING}

The simulated annealing calculation is performed on a honeycomb lattice with $L \times L$ unit cells (or $L \times L \times 2$ sites) with periodic boundary conditions. Most of the computations are done with $L=12,15,20$, but sometimes other $L$ is used when the ground-state spin configuration is not obvious. The procedure of simulated annealing is outlined as follows. In the beginning, we generate a totally random spin configuration on the honeycomb lattice and define a "temperature" parameter $T$. We randomly select a site on the honeycomb lattice and propose a random orientation for the spin on that site. Next, we calculate the difference in energy and accept the change with the probability $\min \{1, \exp (-\Delta E / T)\}$. This step is repeated for $\approx 10^{7}$ times at a fixed $T$, which is then decreased gradually. Once $T<T_{\mathrm{c}}$ for some critical temperature $\approx 10^{-8}|K|$, we update the spin at site $i$ deterministically by aligning it in the direction of the local field [32] defined as

$$
\mathbf{B}_{i}=-\sum_{j} H_{i j} \mathbf{S}_{j}+\mathbf{h},
$$

where $H_{i j}$ is the three-dimensional matrix that encodes the interaction between the spins at $i$ and $j$. We ensure that the energy converges and the spin configuration becomes stable at the end of the iterations. If the sublattice structure of a magnetic order is known (see Ref. [34]), we can carry out the above procedure for a small number of spins and calculate the energy to very high precision. This allows us to better determine the phase boundary between competing orders.

In simulated annealing calculations, like any numerical simulation of a physical system, we have to work with finitesize systems. A valid concern is thus whether there are still ordering patterns that can be realized in the thermodynamic limit but cannot be captured by our simulations. We attempt to reduce such finite-size effects through various strategies. The most obvious and important one is to choose a considerably 
large system. For instance, the $20 \times 20$ unit cell lattice we examine in this study is significantly greater than the 24-site cluster used in exact diagonalization. For a sufficiently large system, if some ordering pattern (e.g., incommensurate order) that does not fit the size or geometry of the lattice is stabilized, then we will see it occupy a finite portion of the large system, if not over the entire system. In other words, there will still be visible traces of such an ordering pattern in a large system if it is the real ground state. Apart from the real space spin configuration, we also examine the static spin structure factor (see Ref. [34]) which peaks at the ordering wave vector. There will be signals at incommensurate wave vectors if some incommensurate order is (partially) realized.

Two other strategies to improve the accuracy of our phase diagrams are described as follows. (i) If competing magnetic orders show up simultaneously, we calculate the energies of these orders separately and compare them. This is how we know, for instance, that the 6-site and 18-site orders are degenerate at zero field in the $K \Gamma$ model. (ii) The parameter space under study is sampled densely, especially in regions where two or more magnetic orders are competing (e.g., phase boundaries). We use a high resolution up to $\Delta \Gamma=0.01$ and $\Delta h=0.01$ for the parameter space under investigation. Different initial conditions are tried if further ambiguity arises. Having taken these steps, we strongly believe that our phase diagrams in Figs. 1 and 2 have very high levels of confidence.

\section{APPENDIX B: DETAILS OF LINEAR SPIN WAVE THEORY}

The content in this section is mainly derived from Ref. [35]. For each sublattice $i$ in the magnetic unit cell, we first choose a local coordinates system in which the spin $\mathbf{S}_{i}$ aligns in the $z$ direction. The amounts to a change of basis characterized by the rotation matrix

$$
R_{i}=\left(\begin{array}{ccc}
\cos \theta_{i} \cos \phi_{i} & -\sin \phi_{i} & \sin \theta_{i} \cos \phi_{i} \\
\cos \theta_{i} \sin \phi_{i} & \cos \phi_{i} & \sin \theta_{i} \sin \phi_{i} \\
-\sin \theta_{i} & 0 & \cos \theta_{i}
\end{array}\right),
$$

where $\theta_{i}$ and $\phi_{i}$ are the two angles parametrizing the orientation of $\mathbf{S}_{i}$ in the cubic $x y z$ coordinates, $\left(S_{i}^{x}, S_{i}^{y}, S_{i}^{z}\right)=$ $S\left(\sin \theta_{i} \cos \phi_{i}, \sin \theta_{i} \sin \phi_{i}, \cos \theta_{i}\right)$. The third column of $R_{i}$ is precisely $\mathbf{S}_{i}$ up to the factor $S$, while the first and second columns are chosen such that the three columns are mutually orthonormal and satisfy the right-hand rule. We define $\mathbf{S}_{i}=$ $R_{i} \tilde{\mathbf{S}}_{i}$. Classically, we have $\tilde{\mathbf{S}}_{i}=(0,0, S)$. Quantum effects on the ordered moments are introduced through spin wave excitations (magnons),

$$
\begin{aligned}
& \tilde{S}_{i}^{z}=S-b_{i}^{\dagger} b_{i}=S-n_{i}, \\
& \tilde{S}_{i}^{x}=\frac{\sqrt{2 S-n_{i}} b_{i}+b_{i}^{\dagger} \sqrt{2 S-n_{i}}}{2} \approx \sqrt{\frac{S}{2}\left(b_{i}+b_{i}^{\dagger}\right),} \\
& \tilde{S}_{i}^{y}=\frac{\sqrt{2 S-n_{i}} b_{i}-b_{i}^{\dagger} \sqrt{2 S-n_{i}}}{2 i} \approx-i \sqrt{\frac{S}{2}}\left(b_{i}-b_{i}^{\dagger}\right),
\end{aligned}
$$

where we have used the linear spin wave approximation that neglects the third and higher order terms in $b_{i}$ in the series expansion of (B2b) and (B2c). Next, we rewrite the spin
Hamiltonian as

$$
H=\sum_{i j} \mathbf{S}_{i}^{\mathrm{T}} H_{i j} \mathbf{S}_{j}-\mathbf{h}^{\mathrm{T}} \sum_{i} \mathbf{S}_{i}=\sum_{i j} \tilde{\mathbf{S}}_{i}^{\mathrm{T}} \tilde{H}_{i j} \tilde{\mathbf{S}}_{j}-\sum_{i} \tilde{\mathbf{h}}_{i}^{\mathrm{T}} \tilde{\mathbf{S}}_{i},
$$

where $\tilde{H}_{i j}=R_{i}^{\mathrm{T}} H_{i j} R_{j}$ and $\tilde{\mathbf{h}}_{i}=R_{i}^{\mathrm{T}} \mathbf{h}$. Representing $\tilde{\mathbf{S}}_{i}$ using (B2a)-(B2c), keeping only terms quadratic in $b_{i}$, and performing a Fourier transform

$$
b_{\mathbf{k}, s}=\frac{1}{\sqrt{N}} \sum_{i} b_{i, s} e^{\mathbf{k} \cdot \mathbf{R}_{i}}
$$

where, from now on, $i$ denotes the position in the Bravais lattice defined by the translational symmetries of the magnetic order, $s$ denotes the sublattice in the magnetic unit cell, and $N$ is the total number of magnetic unit cells, we then obtain the spin wave Hamiltonian in momentum space

$$
H=\sum_{\mathbf{k}} \Psi_{\mathbf{k}}^{\dagger} \mathrm{D}_{\mathbf{k}} \Psi_{\mathbf{k}}
$$

where $\Psi_{\mathbf{k}}=\left(b_{\mathbf{k}, 1}, \ldots, b_{\mathbf{k}, \mathcal{N}}, b_{-\mathbf{k}, 1}^{\dagger}, \ldots, b_{-\mathbf{k}, \mathcal{N}}^{\dagger}\right)$ and $\mathcal{N}$ is the total number of sublattices in the magnetic unit cell. $D_{\mathbf{k}}$ is a $2 \mathcal{N}$-dimensional matrix of the form

$$
D_{\mathbf{k}}=\left(\begin{array}{cc}
A_{\mathbf{k}} & B_{\mathbf{k}} \\
B_{-\mathbf{k}}^{*} & A_{-\mathbf{k}}^{\mathrm{T}}
\end{array}\right),
$$

where $A_{\mathbf{k}}$ and $B_{k}$ are $\mathcal{N}$-dimensional matrices. To obtain the spin wave dispersion, we diagonalize $D_{\mathbf{k}}$ by a Bogoliubov transformation in order to to preserve the canonical commutation relation of the bosons,

$$
\mathrm{T}_{\mathbf{k}}^{\dagger} \mathrm{D}_{\mathbf{k}} \mathrm{T}_{\mathbf{k}}=\mathcal{E}_{\mathbf{k}}, \mathrm{T}_{\mathbf{k}} \sigma^{3} \mathrm{~T}_{\mathbf{k}}^{\dagger}=\sigma^{3},
$$

where $\mathcal{E}_{\mathbf{k}}=\operatorname{diag}\left(\varepsilon_{\mathbf{k}, 1}, \ldots, \varepsilon_{\mathbf{k}, \mathcal{N}}, \varepsilon_{-\mathbf{k}, 1}, \ldots, \varepsilon_{-\mathbf{k}, \mathcal{N}}\right)$ and $\sigma^{3}$ is a diagonal matrix with the first $\mathcal{N}$ entries equal to 1 and the last $\mathcal{N}$ entries equal to -1 . The average reduction of ordered moments (B2a) at temperature $T$ can be calculated from the matrix elements of the Bogoliubov transformation,

$$
\begin{gathered}
\frac{1}{N_{\text {site }}} \sum_{i s}\left\langle b_{i s}^{\dagger} b_{i s}\right\rangle=\frac{1}{N \mathcal{N}} \sum_{\mathbf{k}} \sum_{m, n=1}^{\mathcal{N}}\left\{\mathrm{T}_{\mathbf{k}}^{*}(m, n) \mathrm{T}_{\mathbf{k}}(m, n) f_{\mathrm{BE}}\left(\varepsilon_{\mathbf{k}, n}\right)\right. \\
\left.+\mathrm{T}_{\mathbf{k}}^{*}(m, n+\mathcal{N}) \mathrm{T}_{\mathbf{k}}(m, n+\mathcal{N})\left[1+f_{\mathrm{BE}}\left(\varepsilon_{-\mathbf{k}, n}\right)\right]\right\}, \quad(\mathrm{B} 8)
\end{gathered}
$$

where $f_{\mathrm{BE}}$ is the Bose-Einstein distribution,

$$
f_{\mathrm{BE}}(\varepsilon)=\frac{1}{e^{\varepsilon / T}-1} .
$$

\section{APPENDIX C: CALCULATION OF THERMAL HALL CONDUCTIVITY}

We explain the various symbols that appear in the formula (3) for the calculation of the thermal Hall conductivity [38,39]. $n$ is the magnon band index that runs from 1 to $\mathcal{N}$. The function $c_{2}$ is given by

$$
\begin{aligned}
c_{2}(x) & =\int_{0}^{x} d t\left(\ln \frac{1+t}{t}\right)^{2} \\
& =(1+x)\left(\ln \frac{1+x}{x}\right)^{2}-(\ln x)^{2}-2 \operatorname{Li}_{2}(-x),
\end{aligned}
$$


where $\mathrm{Li}_{2}$ is the dilogarithm. $f_{\mathrm{BE}}$ is the Bose-Einstein distribution as defined in (B9). $\Omega_{\mathbf{k}, n}$ is the Berry curvature defined as

$$
\Omega_{\mathbf{k}, n}=i \epsilon_{\mu \nu}\left[\sigma^{3} \frac{\partial \mathrm{T}_{\mathbf{k}}^{\dagger}}{\partial k_{\mu}} \sigma^{3} \frac{\partial \mathrm{T}_{\mathbf{k}}}{\partial k_{\nu}}\right]_{n n},
$$

where $\sigma^{3}$ and $T_{\mathbf{k}}$ are defined as in (B7). For the calculation of the total volume $V$ of the system, we use the interplane distance $5.72 \AA$ between the honeycomb layers in $\alpha-\mathrm{RuCl}_{3}$ $[19,20]$. The exact value of the in-plane lattice constant does not enter the calculation explicitly because, while $1 / \mathrm{V}$ contributes two inverse factors of it, $\Omega_{\mathbf{k}, n}$ contributes two factors, so they cancel out. When performing the summation over momenta in (3), we partition the first Brillouin zone (of the magnetic order) evenly such that it contains a total number of $L \times L \mathbf{k}$ points. We check the convergence of $\kappa_{x y}$ with increasing $L$ up to $L=800$. We also ensure that the Chern number of each magnon band,

$$
C_{n}=\frac{1}{2 \pi} \sum_{\mathbf{k}} \frac{(2 \pi)^{2}}{A} \Omega_{\mathbf{k}, n},
$$

where $A$ is the total area of the system, converges to an integer with increasing $L$.
[1] Y. Zhou, K. Kanoda, and T.-K. Ng, Quantum spin liquid states, Rev. Mod. Phys. 89, 025003 (2017).

[2] H. Takagi, T. Takayama, G. Jackeli, G. Khaliullin, and S. E. Nagler, Concept and realization of Kitaev quantum spin liquids, Nat. Rev. Phys. 1, 264 (2019).

[3] W. Witczak-Krempa, G. Chen, Y. B. Kim, and L. Balents, Correlated quantum phenomena in the strong spin-orbit regime, Annu. Rev. Condens. Matter Phys. 5, 57 (2014).

[4] J. G. Rau, E. K.-H. Lee, and H.-Y. Kee, Spin-orbit physics giving rise to novel phases in correlated systems: Iridates and related materials, Annu. Rev. Condens. Matter Phys. 7, 195 (2016).

[5] A. Kitaev, Anyons in an exactly solved model and beyond, Ann. Phys. 321, 2 (2006).

[6] G. Jackeli and G. Khaliullin, Mott Insulators in the Strong SpinOrbit Coupling Limit: From Heisenberg to a Quantum Compass and Kitaev Models, Phys. Rev. Lett. 102, 017205 (2009).

[7] J. Chaloupka, G. Jackeli, and G. Khaliullin, Kitaev-Heisenberg Model on a Honeycomb Lattice: Possible Exotic Phases in Iridium Oxides $\mathrm{A}_{2} \mathrm{IrO}_{3}$, Phys. Rev. Lett. 105, 027204 (2010).

[8] V. M. Katukuri, S. Nishimoto, V. Yushankhai, A. Stoyanova, H. Kandpal, S. Choi, R. Coldea, I. Rousochatzakis, L. Hozoi, and J. van den Brink, Kitaev interactions between $j=1 / 2$ moments in honeycomb $\mathrm{Na}_{2} \mathrm{IrO}_{3}$ are large and ferromagnetic: Insights from $a b$ initio quantum chemistry calculations, New J. Phys. 16, 013056 (2014).

[9] T. Takayama, A. Kato, R. Dinnebier, J. Nuss, H. Kono, L. S. I. Veiga, G. Fabbris, D. Haskel, and H. Takagi, Hyperhoneycomb Iridate $\beta-\mathrm{Li}_{2} \mathrm{IrO}_{3}$ as a Platform for Kitaev Magnetism, Phys. Rev. Lett. 114, 077202 (2015).

[10] K. W. Plumb, J. P. Clancy, L. J. Sandilands, V. V. Shankar, Y. F. $\mathrm{Hu}, \mathrm{K} . \mathrm{S}$. Burch, H.-Y. Kee, and Y.-J. Kim, $\alpha-\mathrm{RuCl}_{3}$ : A spinorbit assisted Mott insulator on a honeycomb lattice, Phys. Rev. B 90, 041112(R) (2014).

[11] J. G. Rau, E. K.-H. Lee, and H.-Y. Kee, Generic Spin Model for the Honeycomb Iridates beyond the Kitaev Limit, Phys. Rev. Lett. 112, 077204 (2014).

[12] X. Liu, T. Berlijn, W.-G. Yin, W. Ku, A. Tsvelik, Y.-J. Kim, H. Gretarsson, Y. Singh, P. Gegenwart, and J. P. Hill, Long-range magnetic ordering in $\mathrm{Na}_{2} \mathrm{IrO}_{3}$, Phys. Rev. B 83, 220403(R) (2011).

[13] F. Ye, S. Chi, H. Cao, B. C. Chakoumakos, J. A. FernandezBaca, R. Custelcean, T. F. Qi, O. B. Korneta, and G. Cao, Direct evidence of a zigzag spin-chain structure in the honeycomb lattice: A neutron and $\mathrm{x}$-ray diffraction investigation of singlecrystal $\mathrm{Na}_{2} \mathrm{IrO}_{3}$, Phys. Rev. B 85, 180403(R) (2012).

[14] J. A. Sears, M. Songvilay, K. W. Plumb, J. P. Clancy, Y. Qiu, Y. Zhao, D. Parshall, and Y.-J. Kim, Magnetic order in $\alpha-\mathrm{RuCl}_{3}$ : A honeycomb-lattice quantum magnet with strong spin-orbit coupling, Phys. Rev. B 91, 144420 (2015).

[15] R. D. Johnson, S. C. Williams, A. A. Haghighirad, J. Singleton, V. Zapf, P. Manuel, I. I. Mazin, Y. Li, H. O. Jeschke, R. Valentí, and R. Coldea, Monoclinic crystal structure of $\alpha-\mathrm{RuCl}_{3}$ and the zigzag antiferromagnetic ground state, Phys. Rev. B 92, 235119 (2015).

[16] S. M. Winter, K. Riedl, D. Kaib, R. Coldea, and R. Valentí, Probing $\alpha-\mathrm{RuCl}_{3}$ Beyond Magnetic Order: Effects of Temperature and Magnetic Field, Phys. Rev. Lett. 120, 077203 (2018).

[17] A. Banerjee, P. Lampen-Kelley, J. Knolle, C. Balz, A. A. Aczel, B. Winn, Y. Liu, D. Pajerowski, J. Yan, C. A. Bridges, A. T. Savici, B. C. Chakoumakos, M. D. Lumsden, D. A. Tennant, R. Moessner, D. G. Mandrus, and S. E. Nagler, Excitations in the field-induced quantum spin liquid state of $\alpha-\mathrm{RuCl}_{3}$, npj Quantum Mater. 3, 8 (2018).

[18] C. Balz, P. Lampen-Kelley, A. Banerjee, J. Yan, Z. Lu, X. Hu, S. M. Yadav, Y. Takano, Y. Liu, D. A. Tennant, M. D. Lumsden, D. Mandrus, and S. E. Nagler, Finite field regime for a quantum spin liquid in $\alpha-\mathrm{RuCl}_{3}$, Phys. Rev. B 100, 060405(R) (2019).

[19] Y. Kasahara, K. Sugii, T. Ohnishi, M. Shimozawa, M. Yamashita, N. Kurita, H. Tanaka, J. Nasu, Y. Motome, T. Shibauchi, and Y. Matsuda, Unusual Thermal Hall Effect in a Kitaev Spin Liquid Candidate $\alpha-\mathrm{RuCl}_{3}$, Phys. Rev. Lett. 120, 217205 (2018).

[20] Y. Kasahara, T. Ohnishi, Y. Mizukami, O. Tanaka, S. Ma, K. Sugii, N. Kurita, H. Tanaka, J. Nasu, Y. Motome, T. Shibauchi, and Y. Matsuda, Majorana quantization and half-integer thermal quantum Hall effect in a Kitaev spin liquid, Nature (London) 559, 227 (2018).

[21] H.-S. Kim and H.-Y. Kee, Crystal structure and magnetism in $\alpha-\mathrm{RuCl}_{3}$ : An ab initio study, Phys. Rev. B 93, 155143 (2016).

[22] S. M. Winter, Y. Li, H. O. Jeschke, and R. Valentí, Challenges in design of Kitaev materials: Magnetic interactions from competing energy scales, Phys. Rev. B 93, 214431 (2016).

[23] W. Wang, Z.-Y. Dong, S.-L. Yu, and J.-X. Li, Theoretical investigation of magnetic dynamics in $\alpha-\mathrm{RuCl}_{3}$, Phys. Rev. B 96, 115103 (2017). 
[24] A. Catuneanu, Y. Yamaji, G. Wachtel, Y. B. Kim, and H.-Y. Kee, Path to stable quantum spin liquids in spin-orbit coupled correlated materials, npj Quantum Mater. 3, 23 (2018).

[25] J. Rusnačko, D. Gotfryd, and J. Chaloupka, Kitaev-like honeycomb magnets: Global phase behavior and emergent effective models, Phys. Rev. B 99, 064425 (2019).

[26] Y.-F. Jiang, T. P. Devereaux, and H.-C. Jiang, Field-induced quantum spin liquid in the Kitaev-Heisenberg model and its relation to $\alpha-\mathrm{RuCl}_{3}$, Phys. Rev. B 100, 165123 (2019).

[27] J. S. Gordon, A. Catuneanu, E. S. Sørensen, and H.-Y. Kee, Theory of the field-revealed Kitaev spin liquid, Nat. Commun. 10, 2470 (2019).

[28] D. A. S. Kaib, S. M. Winter, and R. Valentí, Kitaev honeycomb models in magnetic fields: Dynamical response and dual models, Phys. Rev. B 100, 144445 (2019).

[29] M. Gohlke, G. Wachtel, Y. Yamaji, F. Pollmann, and Y. B. Kim, Quantum spin liquid signatures in Kitaev-like frustrated magnets, Phys. Rev. B 97, 075126 (2018).

[30] J. Wang, B. Normand, and Z.-X. Liu, One Proximate Kitaev Spin Liquid in the $K-J-\Gamma$ Model on the Honeycomb Lattice, Phys. Rev. Lett. 123, 197201 (2019).

[31] P. Lampen-Kelley, L. Janssen, E. C. Andrade, S. Rachel, J.-Q. Yan, C. Balz, D. G. Mandrus, S. E. Nagler, and M. Vojta, Fieldinduced intermediate phase in $\alpha-\mathrm{RuCl}_{3}$ : Non-coplanar order, phase diagram, and proximate spin liquid, arXiv:1807.06192.

[32] L. Janssen, E. C. Andrade, and M. Vojta, Honeycomb-Lattice Heisenberg-Kitaev Model in a Magnetic Field: Spin Canting, Metamagnetism, and Vortex Crystals, Phys. Rev. Lett. 117, 277202 (2016).

[33] J. G. Rau and H.-Y. Kee, Trigonal distortion in the honeycomb iridates: Proximity of zigzag and spiral phases in $\mathrm{Na}_{2} \mathrm{IrO}_{3}$, arXiv:1408.4811.

[34] See Supplemental Material at http://link.aps.org/supplemental/ 10.1103/PhysRevResearch.2.013014 for details of the magnetic orders.

[35] D. H. Jones, Q. A. Pankhurst, and C. E. Johnson, Spin-wave theory of anisotropic antiferromagnets in applied magnetic fields, J. Phys. C: Solid State Phys. 20, 5149 (1987).

[36] J. Cookmeyer and J. E. Moore, Spin-wave analysis of the lowtemperature thermal Hall effect in the candidate Kitaev spin liquid $\alpha-\mathrm{RuCl}_{3}$, Phys. Rev. B 98, 060412(R) (2018).
[37] H. Katsura, N. Nagaosa, and P. A. Lee, Theory of the Thermal Hall Effect in Quantum Magnets, Phys. Rev. Lett. 104, 066403 (2010).

[38] R. Matsumoto, R. Shindou, and S. Murakami, Thermal Hall effect of magnons in magnets with dipolar interaction, Phys. Rev. B 89, 054420 (2014).

[39] S. Murakami and A. Okamoto, Thermal Hall effect of magnons, J. Phys. Soc. Jpn. 86, 011010 (2017).

[40] L. Wu, A. Little, E. E. Aldape, D. Rees, E. Thewalt, P. LampenKelley, A. Banerjee, C. A. Bridges, J.-Q. Yan, D. Boone, S. Patankar, D. Goldhaber-Gordon, D. Mandrus, S. E. Nagler, E. Altman, and J. Orenstein, Field evolution of magnons in $\alpha-\mathrm{RuCl}_{3}$ by high-resolution polarized terahertz spectroscopy, Phys. Rev. B 98, 094425 (2018).

[41] J. Chaloupka and G. Khaliullin, Hidden symmetries of the extended Kitaev-Heisenberg model: Implications for the honeycomb-lattice iridates $\mathrm{A}_{2} \mathrm{IrO}_{3}$, Phys. Rev. B 92, 024413 (2015).

[42] G. Baskaran, D. Sen, and R. Shankar, Spin-S Kitaev model: Classical ground states, order from disorder, and exact correlation functions, Phys. Rev. B 78, 115116 (2008).

[43] L. Janssen, E. C. Andrade, and M. Vojta, Magnetization processes of zigzag states on the honeycomb lattice: Identifying spin models for $\alpha-\mathrm{RuCl}_{3}$ and $\mathrm{Na}_{2} \mathrm{IrO}_{3}$, Phys. Rev. B 96, 064430 (2017).

[44] A. Banerjee, C. A. Bridges, J.-Q. Yan, A. A. Aczel, L. Li, M. B. Stone, G. E. Granroth, M. D. Lumsden, Y. Yiu, J. Knolle, S. Bhattacharjee, D. L. Kovrizhin, R. Moessner, D. A. Tennant, D. G. Mandrus, and S. E. Nagler, Proximate Kitaev quantum spin liquid behaviour in a honeycomb magnet, Nat. Mater. 15, 733 (2016).

[45] A. Banerjee, J. Yan, J. Knolle, C. A. Bridges, M. B. Stone, M. D. Lumsden, D. G. Mandrus, D. A. Tennant, R. Moessner, and S. E. Nagler, Neutron scattering in the proximate quantum spin liquid $\alpha-\mathrm{RuCl}_{3}$, Science 356, 1055 (2017).

[46] C. Loken, D. Gruner, L. Groer, R. Peltier, N. Bunn, M. Craig, T. Henriques, J. Dempsey, C.-H. Yu, J. Chen, L. J. Dursi, J. Chong, S. Northrup, J. Pinto, N. Knecht, and R. V. Zon, Scinet: Lessons learned from building a power-efficient top-20 system and data centre, J. Phys.: Conf. Ser. 256, 012026 (2010). 